\title{
Screening of flavonoid aglycons' metabolism mediated by the human liver cytochromes P450
}

\author{
GORAN BENKOVIĆ ${ }^{1}$ \\ MIRZA BOJIĆ2,* \\ ŽELJAN MALEŠ ${ }^{3}$ \\ SINIŠA TOMIĆ ${ }^{1}$ \\ ${ }^{1}$ Agency for Medicinal Products \\ and Medical Devices, HR-10000 Zagreb \\ Croatia \\ ${ }^{2}$ University of Zagreb Faculty of \\ Pharmacy and Biochemistry \\ Department of Medicinal Chemistry \\ HR-10000 Zagreb, Croatia \\ ${ }^{3}$ University of Zagreb Faculty of \\ Pharmacy and Biochemistry \\ Department of Pharmaceutical Botany \\ HR-10000 Zagreb, Croatia
}

Accepted July 23, 2019

Published online September 10, 2019

\begin{abstract}
Biological effects of flavonoids have been extensively studied in the last 80 years. As flavonoids represent a rather large group of compounds, data on metabolic biotransformations of these compounds is relatively limited to those well studied. The objective of this study was to screen the metabolism of 30 selected flavonoid aglycons mediated by the most relevant metabolic enzymes, human liver cytochromes P450. For this purpose, in vitro experiments with human liver microsomes and recombinant enzymes were conducted. To evaluate flavonoid's metabolism and structure of the products, high-performance liquid chromatography coupled with high-resolution mass spectrometry was used. Out of 30 flavonoids, 15 were susceptible to oxidative metabolism mediated by cytochromes P450. Dominant reactions were aromatic hydroxylation and $\mathrm{O}$-demethylation, or a combination of these reactions. The dominant enzyme responsible for the observed metabolic reactions is CYP1A2, whereas other human liver cytochromes P450, namely, CYP2C19, CYP2D6, CYP2E1 and CYP3A4, contribute to flavonoid metabolism to a lesser degree. These results, to some extent, contribute to the understanding of the metabolism of constituents found in antioxidant dietary supplements and their possible interactions with other xenobiotics, i.e., medicinal products.
\end{abstract}

Keywords: flavonoids, human liver cytochromes P450, metabolism, HPLC, MS

In 1936 professor Szent-Györgyi isolated a reducing agent, co-factor in reactions of peroxidase and vitamin $C$. This agent was named vitamin $P$ and later on was identified as rutin (1). To this date, more than 4000 different compounds have been identified and classified as flavonoids (2). Flavonoids are low molecular polyphenols containing benzo- $\gamma-$ -pyrone core in their structure (Fig. 1).

A significant number of pharmacological effects of flavonoids has been described, e.g., antithrombotic, antihypertensive, hypolipemic, antimicrobial, antiallergic, antineoplastic,

\footnotetext{
*Correspondence, e-mail: mbojic@pharma.hr
} 
a)<smiles>O=c1ccoc2ccccc12</smiles>

b)<smiles>c1ccc(C2CCc3ccccc3O2)cc1</smiles>

Fig. 1. Structures of: a) benzo- $\gamma$-pyrone, and b) flavonoid core.

anti-inflammatory, and hepatoprotective effect $(1,3)$. Although the amount of available data on their positive health benefits based on in vitro experiments is overwhelming, there are differences between effects observed in vivo and in vitro (4).

To some extent, this is related to the lack of information on flavonoids' metabolism, that has been characterized for a relatively small number of compounds. Major enzymes that contribute to the metabolism of xenobiotics, including flavonoids, are cytochromes P450. Cytochromes P450 participate in the metabolism of nearly $3 / 4$ of all small drugs and other xenobiotics. Most significant cytochrome P450 is 3A4 that participates in the metabolism of over $50 \%$ of xenobiotics, whereas flavonoids are mainly metabolized with cytochrome P450 1A2 (5). Knowing the cytochrome P450 enzymes involved in the metabolism of flavonoids contributes to the understanding of their bioavailability, pharmacokinetics, and consequently pharmacodynamics, as well as potential interactions with other concomitantly used medications.

The data on pharmacokinetics and metabolism of flavonoids is limited (6-20). Research articles on the metabolism of individual flavonoid used in this study are referenced in Table I. These are often limited to observatory studies of induction and inhibition of cytochromes P450 (21, 22). Flavonoids mainly undergo cytochrome P450 reactions of aromatic hydroxylation and $\mathrm{O}$-demethylation (Fig. 2).

Thus, the objective of this research was to determine human liver cytochromes P450 involved in the metabolism of 30 selected flavonoid aglycons. The set used represents most commonly found flavonoid aglycons in plant species rich in polyphenols. For this purpose, experiments were conducted using commercially available human liver microsomes and recombinant cytochromes P450. Metabolism was monitored by high-performance liquid chromatography (HPLC) coupled with diode array detector (DAD) and high-resolution mass spectrometer (MS).

\section{EXPERIMENTAL}

\section{Chemicals}

Methanol and formic acid (LC-MS grade) were purchased from Honeywell Specialty Chemicals Seelze GmbH, Germany, and Merck KGaA, Germany, resp. Ultra-pure water was produced using Arium comfort combined water production system from Sartorius AG, Germany. Flavone, flavanone, 6-hydroxyflavone, 7-hydroxyflavone, chrysin, 3,6-dihydroxyflavone, 3,7-dihydroxyflavone, pinocembrin, tectochrysin, apigenin, galangin, genistein, pinocembrin-7-methylether, naringenin, kaempferol, sakuranetin, catechin, diosmetin, morin, quercetin, hesperetin, isorhamnetin, tamarixetin, rhamnetin, myricetin, and 
G. Benković et al.: Screening of flavonoid aglycons' metabolism mediated by the human liver cytochromes P450, Acta Pharm. 69 (2019) 541-562.

Table I. Structural features of flavonoids used in the screening of cytochromes P450 mediated metabolisma

\begin{tabular}{|c|c|c|c|c|c|c|c|c|c|c|}
\hline No. & Flavonoid & C5 & C6 & $\mathrm{C} 7$ & $\mathrm{C} 8$ & $\mathrm{C} 2^{\prime}$ & $\mathrm{C}^{\prime}$ & $C 4^{\prime}$ & C5 & $\begin{array}{l}\text { Metabolic reaction } \\
\text { (literature source) }^{\mathrm{a}}\end{array}$ \\
\hline & \multicolumn{10}{|c|}{ Flavones $(\mathrm{C} 2-\mathrm{Ph}, \mathrm{C} 2=\mathrm{C} 3, \mathrm{C} 4=\mathrm{O})$} \\
\hline 1 & Acacetin & $\mathrm{OH}$ & $\mathrm{H}$ & $\mathrm{OH}$ & $\mathrm{H}$ & $\mathrm{H}$ & $\mathrm{H}$ & $\mathrm{OCH}_{3}$ & $\mathrm{H}$ & 4'-demethylation (6) \\
\hline 2 & Apigenin & $\mathrm{OH}$ & $\mathrm{H}$ & $\mathrm{OH}$ & $\mathrm{H}$ & $\mathrm{H}$ & $\mathrm{H}$ & $\mathrm{OH}$ & $\mathrm{H}$ & 3'-hydroxylation (7-9) \\
\hline 3 & Diosmetin & $\mathrm{OH}$ & $\mathrm{H}$ & $\mathrm{OH}$ & $\mathrm{H}$ & $\mathrm{H}$ & $\mathrm{OH}$ & $\mathrm{OCH}_{3}$ & $\mathrm{H}$ & \multirow[t]{4}{*}{ 4'-demethylation (10) } \\
\hline 4 & Flavone & $\mathrm{H}$ & $\mathrm{H}$ & $\mathrm{H}$ & $\mathrm{H}$ & $\mathrm{H}$ & $\mathrm{H}$ & $\mathrm{H}$ & $\mathrm{H}$ & \\
\hline 5 & 6-Hydroxyflavone & $\mathrm{H}$ & $\mathrm{OH}$ & $\mathrm{H}$ & $\mathrm{H}$ & $\mathrm{H}$ & $\mathrm{H}$ & $\mathrm{H}$ & $\mathrm{H}$ & \\
\hline 6 & 7-Hydroxyflavone & $\mathrm{H}$ & $\mathrm{H}$ & $\mathrm{OH}$ & $\mathrm{H}$ & $\mathrm{H}$ & $\mathrm{H}$ & $\mathrm{H}$ & $\mathrm{H}$ & \\
\hline 7 & Chrysin & $\mathrm{OH}$ & $\mathrm{H}$ & $\mathrm{OH}$ & $\mathrm{H}$ & $\mathrm{H}$ & $\mathrm{H}$ & $\mathrm{H}$ & $\mathrm{H}$ & \multirow[t]{3}{*}{ 6-hydroxylation (7-9) } \\
\hline 8 & $\begin{array}{l}\text { Chrysin-dimethyl } \\
\text { ether }\end{array}$ & $\mathrm{OCH}_{3}$ & $\mathrm{H}$ & $\mathrm{OCH}_{3}$ & $\mathrm{H}$ & $\mathrm{H}$ & $\mathrm{H}$ & $\mathrm{H}$ & $\mathrm{H}$ & \\
\hline 9 & Luteolin & $\mathrm{OH}$ & $\mathrm{H}$ & $\mathrm{OH}$ & $\mathrm{H}$ & $\mathrm{H}$ & $\mathrm{OH}$ & $\mathrm{OH}$ & $\mathrm{H}$ & \\
\hline 10 & Tangeretin & $\mathrm{OCH}_{3}$ & $\mathrm{OCH}_{3}$ & $\mathrm{OCH}_{3}$ & $\mathrm{OCH}_{3}$ & $\mathrm{H}$ & $\mathrm{H}$ & $\mathrm{OCH}_{3}$ & $\mathrm{H}$ & \multirow[t]{2}{*}{$\begin{array}{l}\text { 4'-demethylation }(7, \\
11,12)\end{array}$} \\
\hline 11 & Techtocrysin & $\mathrm{OH}$ & $\mathrm{H}$ & $\mathrm{OCH}_{3}$ & $\mathrm{H}$ & $\mathrm{H}$ & $\mathrm{H}$ & $\mathrm{H}$ & $\mathrm{H}$ & \\
\hline & \multicolumn{10}{|c|}{ Flavonoles $(\mathrm{C} 2-\mathrm{Ph}, \mathrm{C} 2=\mathrm{C} 3, \mathrm{C} 3-\mathrm{OH}, \mathrm{C} 4=\mathrm{O})$} \\
\hline 12 & 3,6-Dihydroxyflavone & $\mathrm{H}$ & $\mathrm{OH}$ & $\mathrm{H}$ & $\mathrm{H}$ & $\mathrm{H}$ & $\mathrm{H}$ & $\mathrm{H}$ & $\mathrm{H}$ & \multirow{4}{*}{$\begin{array}{l}\text { 4'-hydroxylation } \\
(7,13)\end{array}$} \\
\hline 13 & 3,7-Dihydroxyflavone & $\mathrm{H}$ & $\mathrm{H}$ & $\mathrm{OH}$ & $\mathrm{H}$ & $\mathrm{H}$ & $\mathrm{H}$ & $\mathrm{H}$ & $\mathrm{H}$ & \\
\hline 14 & Galangin & $\mathrm{OH}$ & $\mathrm{H}$ & $\mathrm{OH}$ & $\mathrm{H}$ & $\mathrm{H}$ & $\mathrm{H}$ & $\mathrm{H}$ & $\mathrm{H}$ & \\
\hline 15 & Isorhamnetin & $\mathrm{OH}$ & $\mathrm{H}$ & $\mathrm{OH}$ & $\mathrm{H}$ & $\mathrm{H}$ & $\mathrm{OCH}_{3}$ & $\mathrm{OH}$ & $\mathrm{H}$ & \\
\hline 16 & Kaempferol & $\mathrm{OH}$ & $\mathrm{H}$ & $\mathrm{OH}$ & $\mathrm{H}$ & $\mathrm{H}$ & $\mathrm{H}$ & $\mathrm{OH}$ & $\mathrm{H}$ & \multirow[t]{5}{*}{$\begin{array}{l}\text { 3'-hydroxylation } \\
(7,8)\end{array}$} \\
\hline 17 & Morin & $\mathrm{OH}$ & $\mathrm{H}$ & $\mathrm{OH}$ & $\mathrm{H}$ & $\mathrm{OH}$ & $\mathrm{H}$ & $\mathrm{OH}$ & $\mathrm{H}$ & \\
\hline 18 & Myricetin & $\mathrm{OH}$ & $\mathrm{H}$ & $\mathrm{OH}$ & $\mathrm{H}$ & $\mathrm{H}$ & $\mathrm{OH}$ & $\mathrm{OH}$ & $\mathrm{OH}$ & \\
\hline 19 & Quercetin & $\mathrm{OH}$ & $\mathrm{H}$ & $\mathrm{OH}$ & $\mathrm{H}$ & $\mathrm{H}$ & $\mathrm{OH}$ & $\mathrm{OH}$ & $\mathrm{H}$ & \\
\hline 20 & Rhamnetin & $\mathrm{OH}$ & $\mathrm{H}$ & $\mathrm{OCH}_{3}$ & $\mathrm{H}$ & $\mathrm{H}$ & $\mathrm{OH}$ & $\mathrm{OH}$ & $\mathrm{H}$ & \\
\hline 21 & Tamarixetin & $\mathrm{OH}$ & $\mathrm{H}$ & $\mathrm{OH}$ & $\mathrm{H}$ & $\mathrm{H}$ & $\mathrm{OH}$ & $\mathrm{OCH}_{3}$ & $\mathrm{H}$ & $\begin{array}{l}\text { 4'-demethylation (7, } \\
8 \text { ) }\end{array}$ \\
\hline & \multicolumn{10}{|c|}{ Isoflavones $(\mathrm{C} 2=\mathrm{C} 3, \mathrm{C} 3-\mathrm{Ph}, \mathrm{C} 4=\mathrm{O})$} \\
\hline 22 & Genistein & $\mathrm{OH}$ & $\mathrm{H}$ & $\mathrm{OH}$ & $\mathrm{H}$ & $\mathrm{H}$ & $\mathrm{H}$ & $\mathrm{OH}$ & $\mathrm{H}$ & $\begin{array}{l}\text { 6-hydroxylation, } \\
\text { 8-hydroxylation, } \\
\text { 3'-hydro- } \\
\text { xylation (14-19) } \\
\text { 3'-hydroxylation } \\
(14-19)\end{array}$ \\
\hline 23 & Prunetin & $\mathrm{OH}$ & $\mathrm{H}$ & $\mathrm{OCH}_{3}$ & $\mathrm{H}$ & $\mathrm{H}$ & $\mathrm{H}$ & $\mathrm{OH}$ & $\mathrm{H}$ & 4'-demethylation (15) \\
\hline & \multicolumn{10}{|c|}{ Flavanones $(\mathrm{C} 2-\mathrm{Ph}, \mathrm{C} 2-\mathrm{C} 3, \mathrm{C} 4=\mathrm{O})$} \\
\hline 24 & Flavanone & $\mathrm{H}$ & $\mathrm{H}$ & $\mathrm{H}$ & $\mathrm{H}$ & $\mathrm{H}$ & $\mathrm{H}$ & $\mathrm{H}$ & $\mathrm{H}$ & \multirow{6}{*}{$\begin{array}{l}\text { 4'-demethylation }(7, \\
8,20) \\
\text { 3'-hydroxylation }(7,8)\end{array}$} \\
\hline 25 & Hesperetin & $\mathrm{OH}$ & $\mathrm{H}$ & $\mathrm{OH}$ & $\mathrm{H}$ & $\mathrm{H}$ & $\mathrm{OH}$ & $\mathrm{OCH}_{3}$ & $\mathrm{H}$ & \\
\hline 26 & Naringenin & $\mathrm{OH}$ & $\mathrm{H}$ & $\mathrm{OH}$ & $\mathrm{H}$ & $\mathrm{H}$ & $\mathrm{H}$ & $\mathrm{OH}$ & $\mathrm{H}$ & \\
\hline 27 & Pinocembrin & $\mathrm{OH}$ & $\mathrm{H}$ & $\mathrm{OH}$ & $\mathrm{H}$ & $\mathrm{H}$ & $\mathrm{H}$ & $\mathrm{H}$ & $\mathrm{H}$ & \\
\hline 28 & $\begin{array}{l}\text { Pinocembrin-7- } \\
\text {-methylether }\end{array}$ & $\mathrm{OH}$ & $\mathrm{H}$ & $\mathrm{OCH}_{3}$ & $\mathrm{H}$ & $\mathrm{H}$ & $\mathrm{H}$ & $\mathrm{H}$ & $\mathrm{H}$ & \\
\hline 29 & Sakuranetin & $\mathrm{OH}$ & $\mathrm{H}$ & $\mathrm{OCH}_{3}$ & $\mathrm{H}$ & $\mathrm{H}$ & $\mathrm{H}$ & $\mathrm{OH}$ & $\mathrm{H}$ & \\
\hline & \multicolumn{10}{|c|}{ Flavanole (C2-Ph, C2-C3, C3-OH, C4=O) } \\
\hline 30 & Catechin & & & $\mathrm{H}$ & $\mathrm{OH}$ & $\mathrm{H}$ & $\mathrm{H}$ & $\mathrm{OH}$ & $\mathrm{OH}$ & \\
\hline
\end{tabular}

a Previous data on most dominant metabolic reactions of individual flavonoids reported under literature column, if available. 

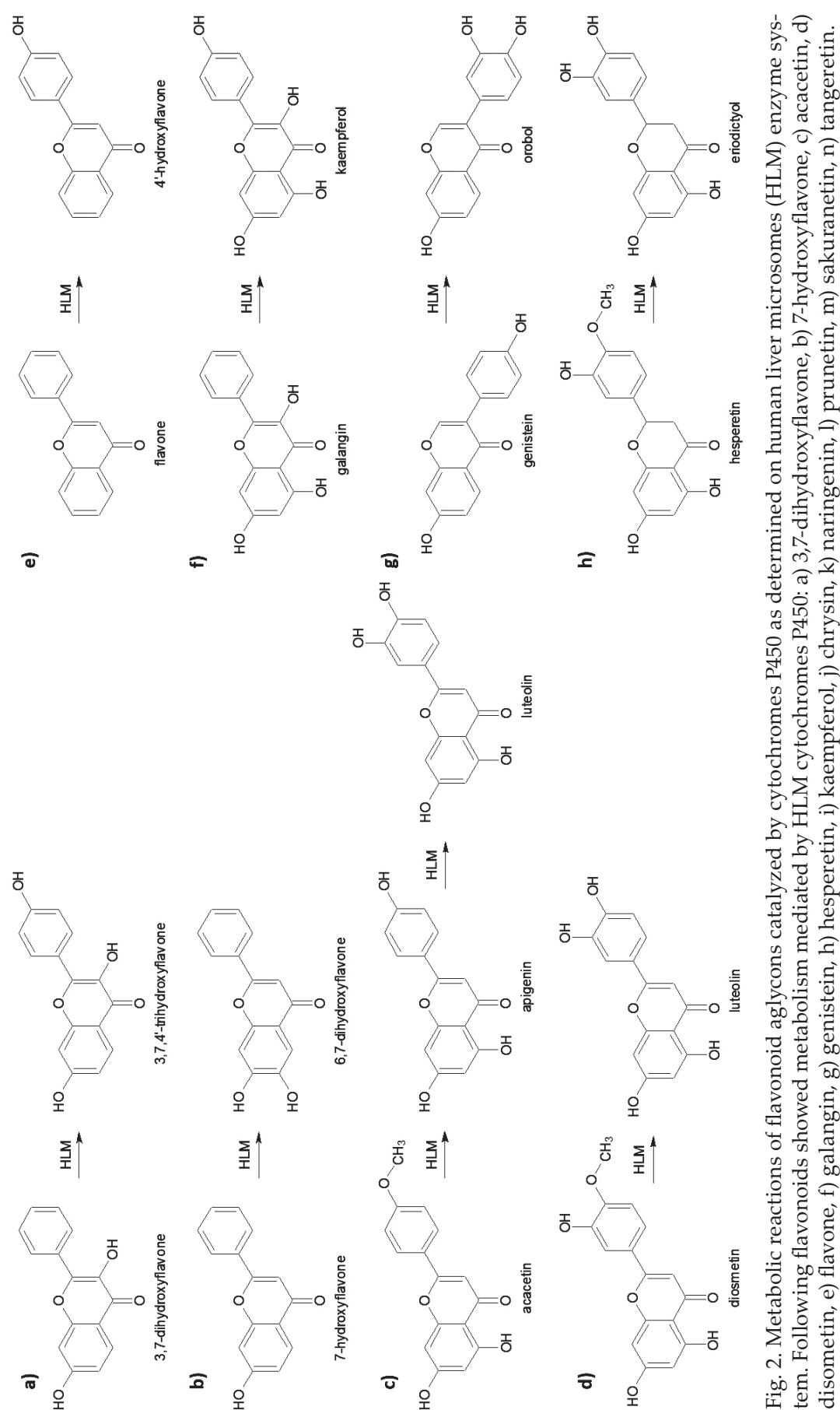

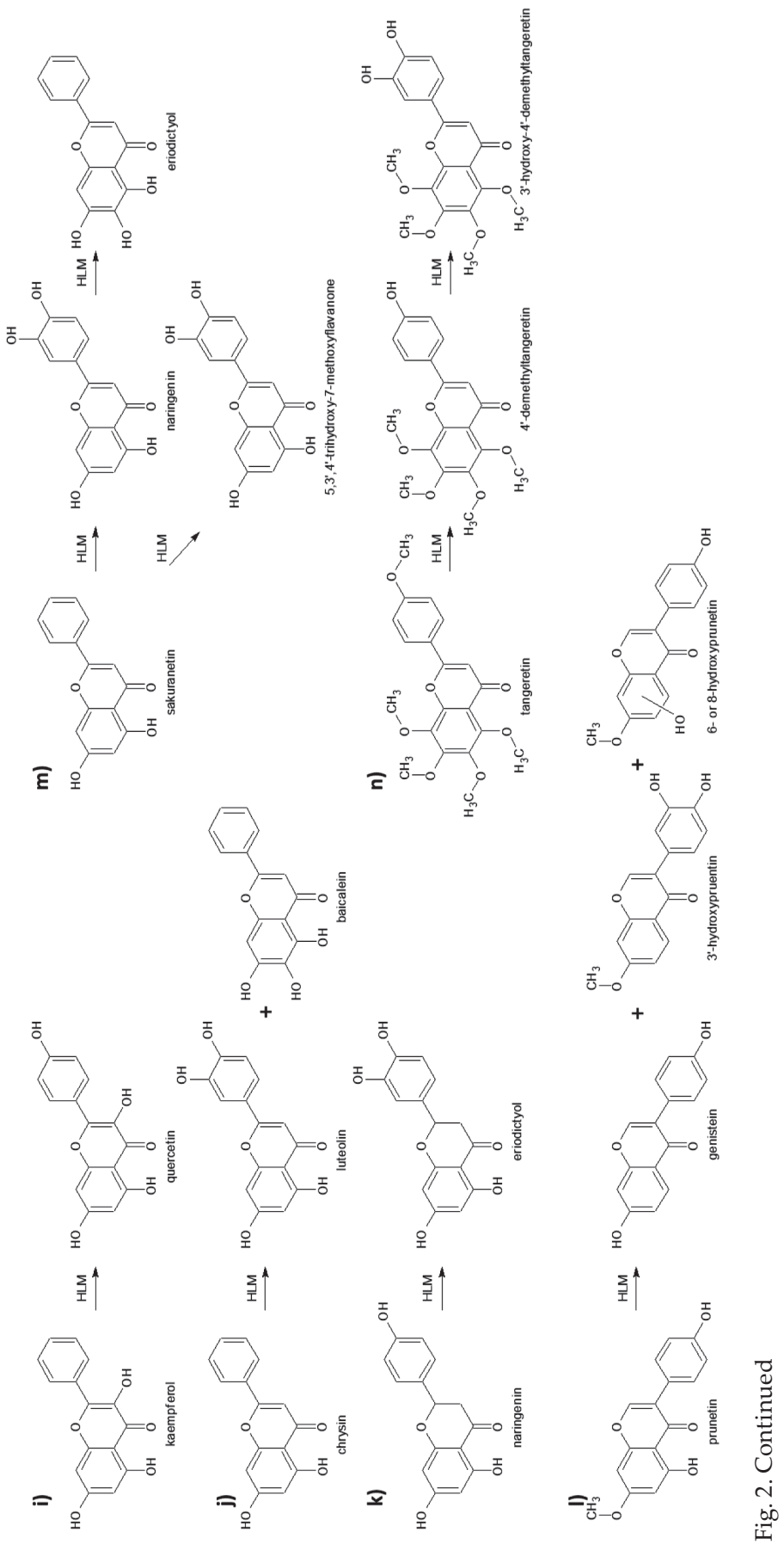
tangeretin were purchased from Extrasynthese, France. 3,7,4'-Trihydroxyflavone, 6,7-dihydroxyflavone, 7,8-dihydroxyflavone, 4'-hydroxyflavone, 2'-hydroxyflavone, 5,3',4'-trihydroxy-7-methoxyflavanone were obtained from TransMIT GmbH, Project Division for Plant Metabolites and Chemicals, Germany, and eriodictyol from Biovit Ltd., Croatia. Human liver microsomes (HLM) and recombinant cytochrome P450 expressed in baculosomes were obtained from Thermo Fisher Scientific, USA. Glucose-6-phosphate (G6P), glucose-6-phosphate dehydrogenase (G6PD) and NADP disodium salt were purchased from Sigma Aldrich, USA. Potassium phosphate and concentrated hydrochloric acid were purchased from Kemika d.d., Croatia. Acetonitrile for chromatography was purchased from Merck KGaA.

\section{Incubation}

Each flavonoid stock solution was individually prepared as $20 \mathrm{mmol} \mathrm{L}^{-1}$ methanol solution. To achieve the desired final concentration during the incubation, an aliquot of the stock solution was transferred to a glass tube and dried to remove the organic solvent. In this way, an appropriate amount of flavonoid was added to the tube and organic solvent was removed from the incubation process as it can interfere with the enzyme activity. Each incubation medium contained 100 pmol of human liver microsomes (HLM, amount relates to cytochrome $\mathrm{P} 450$ enzyme). The $\mathrm{pH}$ of the incubation medium was set to physiological (7.4) using the potassium phosphate buffer solution (final concentration $50 \mathrm{mmol} \mathrm{L}^{-1}$ ). The reaction was initiated by adding $15 \mu \mathrm{L}$ of the generating system (GS) consisting of glucose-6-phosphate, $\mathrm{NADP}^{+}$, and glucose-6-phosphate dehydrogenase in a ratio of 100:50:2 (V/V/V, the final amount in the incubation $15 \%$ ). In the negative control (blind test), $15 \mu \mathrm{L}$ of ultrapure water was added to this step instead of GS. The total volume of each incubation mixture was $100 \mu \mathrm{L}$.

After $15 \mathrm{~min}$ of incubation at $37^{\circ} \mathrm{C}$ in a water bath with mechanical shaking, the reaction was stopped by adding $60 \mu \mathrm{L}$ of a mixture of acetonitrile and $25 \%$ hydrochloric acid solution $(5: 1, V / V)$. The final volume of the incubation mixture, after stopping the reaction, was $160 \mu \mathrm{L}$ and the concentration of flavonoids $125 \mu \mathrm{mol} \mathrm{L}{ }^{-1}$. After stopping the reaction, the incubation mixture was centrifuged at 10,000 rpm for $10 \mathrm{~min}$ in a MiniSpin centrifuge (Eppendorf AG, Germany). The clear supernatant was transferred to the HPLC vial and analyzed by HPLC/MS.

To verify which cytochrome $\mathrm{P} 450$ is involved in the metabolism, specific inhibitors were used in combination with human liver microsomes, or pure recombinant cytochrome P450 was used for analysis replacing HLM. Incubations with recombinant cytochromes P450 were conducted using 3 pmol of the enzyme (CYP1A2, CYP2A6, CYP2C8, CYP2C9, CYP2C19, CYP2D6 or CYP3A4). Specific inhibitors of cytochromes P450 were $1 \mu \mathrm{mol} \mathrm{L}^{-1}$ $\alpha$-naphthoflavone for CYP1A2, $2 \mu \mathrm{mol} \mathrm{L}{ }^{-1}$ methoxalen for CYP2A6, $5 \mu \mathrm{mol} \mathrm{L}^{-1}$ ticlopidin for CYP2B6, $50 \mu \mathrm{mol} \mathrm{L}^{-1}$ quercetin for CYP2C8, $5 \mu \mathrm{mol} \mathrm{L}^{-1}$ sulfaphenazole for CYP2C9, 10 $\mu \mathrm{mol} \mathrm{L}{ }^{-1}$ fluconazole for CYP2C19, $2 \mu \mathrm{mol} \mathrm{L}^{-1}$ ketoconazole for CYP3A4, $2 \mu \mathrm{mol} \mathrm{L}{ }^{-1}$ quinidine for CYP2D6, and $100 \mu \mathrm{mol} \mathrm{L}-1$ 4-methylpyrazole for CYP2E1 (23). The concentration of inhibitor refers to the final concentration of the inhibitor in the incubation mixture.

\section{Liquid chromatography coupled with diode array detector and mass spectrometer}

Liquid chromatography (LC) coupled with diode array detector (DAD) and mass spectrometer (MS) was performed on the Agilent 1200 RR (Agilent Technologies, Germany) LC 
system coupled to Agilent 6530 Accurate Mass Q-TOF HPLC/MS using electrospray ionization (ESI) interface. The column Poroshell EC-C18 $(100 \times 3.0 \mathrm{~mm}$ i.d., $2.7 \mu \mathrm{m}$ particle size, Agilent) used for separation was set at the temperature of $40^{\circ} \mathrm{C}$. The flow rate was set at $0.4 \mathrm{~mL} \mathrm{~min}^{-1}$ and the injection volume was $5 \mu \mathrm{L}$. Mobile phase A consisted of water, methanol and formic acid at a ratio of 93:5:2 $(V / V / V)$ whereas the ratio of the same components in mobile phase $\mathrm{B}$ was 3:95:2 ( $V / V / V)$. The gradient was carried out according to the following scheme: 0 min, $40 \%$ B; 14-15 min, $80 \%$ B; 16-20 min, $40 \%$ B. The DAD was set at $350 \mathrm{~nm}$. Mass spectrometry data collection was performed in MS high-resolution mode, ESI source was set to the positive ionization mode and mass spectra were recorded in $\mathrm{m} / \mathrm{z}$ range of 100 to 1000. Operating conditions of the MS detector were as follows: drying gas flow $8 \mathrm{~L} \mathrm{~min}^{-1}$ at $275.8 \mathrm{kPa}$ nebulizer gas pressure and drying gas temperature at $200^{\circ} \mathrm{C}$, sheath gas flow was $11 \mathrm{~L} \mathrm{~min}^{-1}$ with sheath gas temperature set at $300^{\circ} \mathrm{C}$. The time-of-flight (TOF) analyzer was used with the following settings: the voltage of the fragment, skimmer and the octapol were 175, 65 and $750 \mathrm{~V}$, resp. All data were collected and processed using Agilent MassHunter Workstation Software Program (Agilent Technologies, USA). Identification of the metabolites was based on a comparison of exact molecular mass and time of metabolite retention relative to the reference standards. Quantification of the detected metabolites was performed on the basis of the signals measured by the UV detector.

\section{RESULTS AND DISCUSSION}

\section{Development of the HPLC/MS method for the identification and determination of flavonoid metabolites}

Development of HPLC/MS method for identification and determination of the metabolites of selected flavonoid aglycons was based on the optimization of the HPLC method used for the analysis of phenolic acids and flavonoids in various wine and propolis samples, published and described in the literature $(24,25)$. The method was chosen primarily because of its wide separation window and mobile phase composition suitable for analysis with the MS detector.

The TOF analyzer allowed accurate mass determination and calculation of the molecular formula for the detected ions of flavonoids based on their specific isotopic patterns, enabling simple identification in conjugation with the retention time. If metabolite was not commercially available, MS/MS spectra were recorded enabling determination of the ring in the flavonoid skeleton that undergoes biotransformation. Possible site of reaction could be elucidated, although the exact structure of unknown metabolite could not be established solely on the basis of MS and MS/MS data. This is in accordance with the previously reported cases of Tsimogiannis et al. (26) and Cuyckens and Claeys (27).

Fig. 3 shows the methodology of identification and determination of flavonoid metabolites using the example of aromatic hydroxylation to which apigenin is susceptible, generating luteolin as a metabolite.

Screening of selected flavonoid aglycons that underwent metabolism mediated by human liver cytochromes P450 was performed using HLM. Biotransformation was observed in 15 out of 30 analyzed flavonoids (Table II). Detected metabolites were generated by reactions of aromatic hydroxylation and $\mathrm{O}$-demethylation, or a combination of these reactions. 
a)

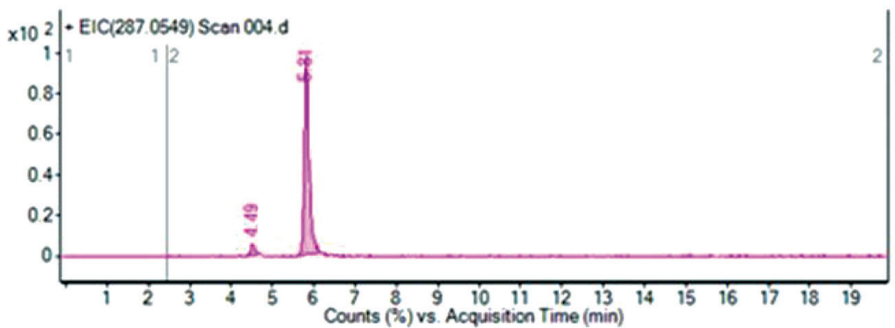

b)

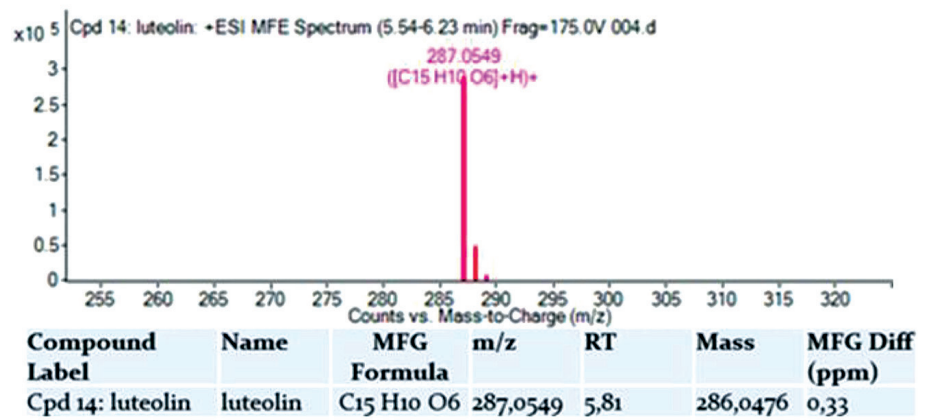

c)

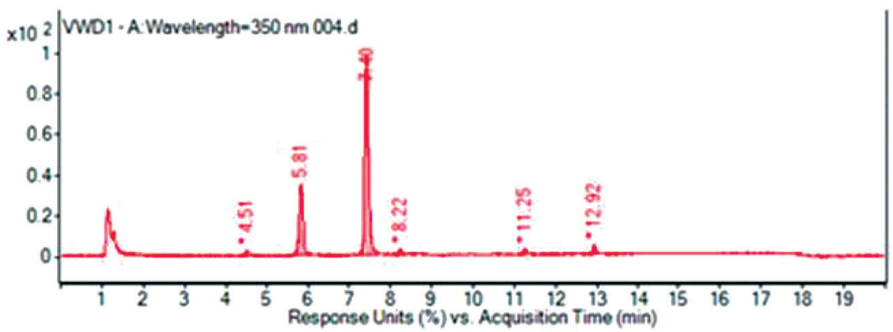

Fig. 3. The methodology of identification and determination of metabolites. Data used for detection of selected flavonoids and their metabolites: retention time, exact mass, molecular formula. An example of an apigenin metabolite is given in the figure. The chromatogram in c) refers to the dominant metabolite with a retention time of $5.81 \mathrm{~min}$. In b) the peak mass spectrum is shown at $5.81 \mathrm{~min}$, and in a) the chromatogram of the separated ion corresponding to the same retention time. Based on comparison with pure standard data stored in the database, an unknown metabolite identified as luteolin.

In the incubations with 6-hydroxyflavone, a mass change of $2.014545 \mathrm{~g} \mathrm{~mol}^{-1}$ was recorded and the molecular formula $\mathrm{C}_{15} \mathrm{H}_{12} \mathrm{O}_{3}$ was calculated on the basis of MS data. These data indicate the formation of dihydroxychalcone by the opening of the ring $\mathrm{C}$ (cleavage of the bond O1-C2, see Fig. 2). The change in mass of -15.99491 was also observed, and based on MS data calculated molecular formula was $\mathrm{C}_{15} \mathrm{H}_{12} \mathrm{O}_{2}$. This indicates degradation (opening) of the ring $\mathrm{C}$ and the formation of monohydroxychalcone. Both of these reactions were observed in incubations without the addition of a generating system, indicating a non-enzymatic reaction. Since the same changes observed in the incubations with the 
added generating system could not be unequivocally attributed to the action of the cytochrome P450 enzyme, and the other changes attributable to the cytochrome P450 activity were not related to the original flavonoid 6-hydroxyflavone, the 6-hydroxyflavone results were not taken into further consideration.

\section{Determination of flavonoid metabolism mediated by human liver microsomes}

In the incubations of 3,7-dihydroxyflavone with human liver microsomes, the formation of hydroxylated metabolites was detected (Fig. 2a). The main product was identified as 3,7,4'-trihydroxyflavone. The second product, present in a much smaller amount, due to the lack of commercially available standard, could not be identified, but, based on the MS and MS/MS data it was further characterized as the A ring hydroxylated product (either at the position 6 or 8 ).

In the incubations of 7-hydroxyfavone with human liver microsomes, two metabolites were detected - hydroxylated products at positions 6 and 8 . The main product was identified as 6,7-dihydroxyflavone (Fig. 2b), and the other product, present in much less quantity, was identified as 7,8-dihydroxyflavone.

Two metabolites of acacetin generated by human liver microsomes were detected: apigenin and luteolin (Fig. 2c). The finding of apigenin and luteolin as metabolism products indicates the sequential formation of the metabolites by the action of cytochromes P450. First, the demethylation at the position $4^{\prime}$ of ring B occurred, generating apigenin which is subsequently hydroxylated at the position 3' of ring B producing luteolin. Apigenin is the most abundant metabolic product, indicating that the acacetin demethylation is the major metabolic pathway catalyzed by cytochromes P450. Similarly, in the incubations of apigenin with human liver microsomes, the metabolite identified was luteolin (Fig. 2c).

Disometin is metabolized to luteolin in the incubations with human liver microsomes as it undergoes $\mathrm{O}$-demethylation at 4'-position of the B ring (Fig. 2d).

In the incubation of flavone with human liver microsomes, metabolite identified as 4'-hydroxyflavone was detected using a UV detector (Fig. 2e). Additional hydroxylated products with different elution times were detected. However, they were generated in negligible quantities. Among them, 6-hydroxyflavone and 7-hydroxyflavone have been identified.

Galangin was metabolized to kaempferol indicating aromatic hydroxylation of galangin at position $4^{\prime}$ of ring B as a major metabolic pathway (Fig. 2f).

In the incubation of genistein with human liver microsomes, orobol was detected as a metabolite (Fig. 2g). The finding of orobol as a metabolic product points to the aromatic hydroxylation of the genistein substrate at position $3^{\prime}$ of the ring $\mathrm{B}$.

When hesperetin was incubated with human liver microsomes the formation of a metabolite identified as eriodictyol was observed (Fig. 2h). Thus, a major metabolic reaction was $\mathrm{O}$-demethylation of the hesperetin substrate at position $4^{\prime}$ of the ring $\mathrm{B}$.

In the incubation of kaempferol with human liver microsomes (HLM), quercetin was identified (Fig. 2i). The finding of quercetin as a metabolic product indicates aromatic hydroxylation of kaempferol at position $3^{\prime}$ of the ring B as the major metabolic reaction.

Chrysin generated two metabolites, baicalein and luteolin (Fig. 2j). Both products were generated by aromatic hydroxylation: at position 6 of ring A (baicalein) and double 
aromatic hydroxylation of ring B at positions $3^{\prime}$ and $4^{\prime}$ (luteolin). Although dihydroxylated product (luteolin) was observed, monohydroxylated products were not found (e.g., apigenin), not even in traces. Thus, chrysin biotransformation to luteolin included two consecutive hydroxylation reactions without the release of monohydroxylated products from the enzyme. In all previous cases where a combined reaction (double hydroxylation or demethylation with hydroxylation) was observed, it was found to be sequentially carried out over a single hydroxylated or demethylated intermediate. Both metabolites were represented in approximately equal amounts indicating that none of these metabolic pathways catalyzed by cytochrome P450 is favored.

The absence of oxidative metabolism of luteolin was also observed by Nielsen et al. (7), although it should be emphasized that the authors in this experiment used rat liver microsomes while in our study human liver microsomes were used.

In the incubation of naringenin with human liver microsomes, eriodictyol was identified as a metabolite (Fig. 2k). Eriodictyol was generated by aromatic hydroxylation of the naringenin at the position $3^{\prime}$ of the ring $\mathrm{B}$.

Prunetin was transformed into three metabolites when incubated with human liver microsomes (Fig. 2l). The most abundant metabolite was identified as genistein, suggesting that $O$-demethylation is a preferred metabolic pathway. In approximately equal and slightly smaller quantities compared to genistein, two aromatic hydroxylated products were generated by separate pathways, one by hydroxylation at position 3' of B ring (known as santal), and the other at position 6 of the ring A.

In the incubations of sakuranetin with human liver microsomes the formation of three metabolites was observed (Fig. $2 \mathrm{~m}$ ). The finding of naringenin and eriodictyol as metabolic products indicates the sequential formation of the metabolite by the action of cytochrome P450. First, the parent flavonoid sakuranetin is demethylated at the position 7 of the ring $\mathrm{A}$ to form naringenin, and then naringenin is hydroxylated at the position $3^{\prime}$ of the ring B, thereby producing eriodictyol. The third product of the sakuranetin metabolism was formed by aromatic hydroxylation at the position $3^{\prime}$ of the ring B, i.e., 5, 3', $4^{\prime}$-trihydroxy-7-methoxyflavanone was formed.

Tangeretin generated more than 10 metabolites in the incubations with human liver microsomes. The largest number of detected metabolites refers to single and double demethylated tangeretin derivatives in various combinations and at different positions on the rings $\mathrm{A}$ and $\mathrm{B}$. Most of these metabolites could not be identified because commercially available standards of potential metabolites were not available. The absence of single demethylation at ring $\mathrm{A}$, and the absence of double demethylation, could be determined solely by LC-MS and LC-MS/MS analysis. Therefore, these products were characterized up to the level of reaction type that was catalyzed by cytochrome P450 under experimental conditions used. However, the two major metabolites, most commonly found in tangeretin incubations with HLM, were indirectly identified based on data obtained by LC-MS/MS analysis (Fig. 2n). MS/MS spectra showed a mass loss of $14.01564 \mathrm{~g} \mathrm{~mol}^{-1}$ on the ring B with $t_{\mathrm{R}}$ of $9.52 \mathrm{~min}$. Since only one methoxy group at position $4^{\prime}$ is present in the tangeretin ring structure $B$, this metabolite is characterized as a $4^{\prime}$-demethylated tangeretin derivative, i.e., $4^{\prime}$-hydroxy-5,6,7,8-tetramethoxyflavone. Similarly, the structure of the second most commonly found metabolite detected at $t_{\mathrm{R}}=7.83$ min showed a mass increase of $1.9799 \mathrm{~g} \mathrm{~mol}^{-1}$ that is located on ring B (based on MS/MS analysis), indicating demethylation at the $4^{\prime}$ position and hydroxylation at the positions $2^{\prime}$ or $3^{\prime}$. Based on the literature data (Table I), 


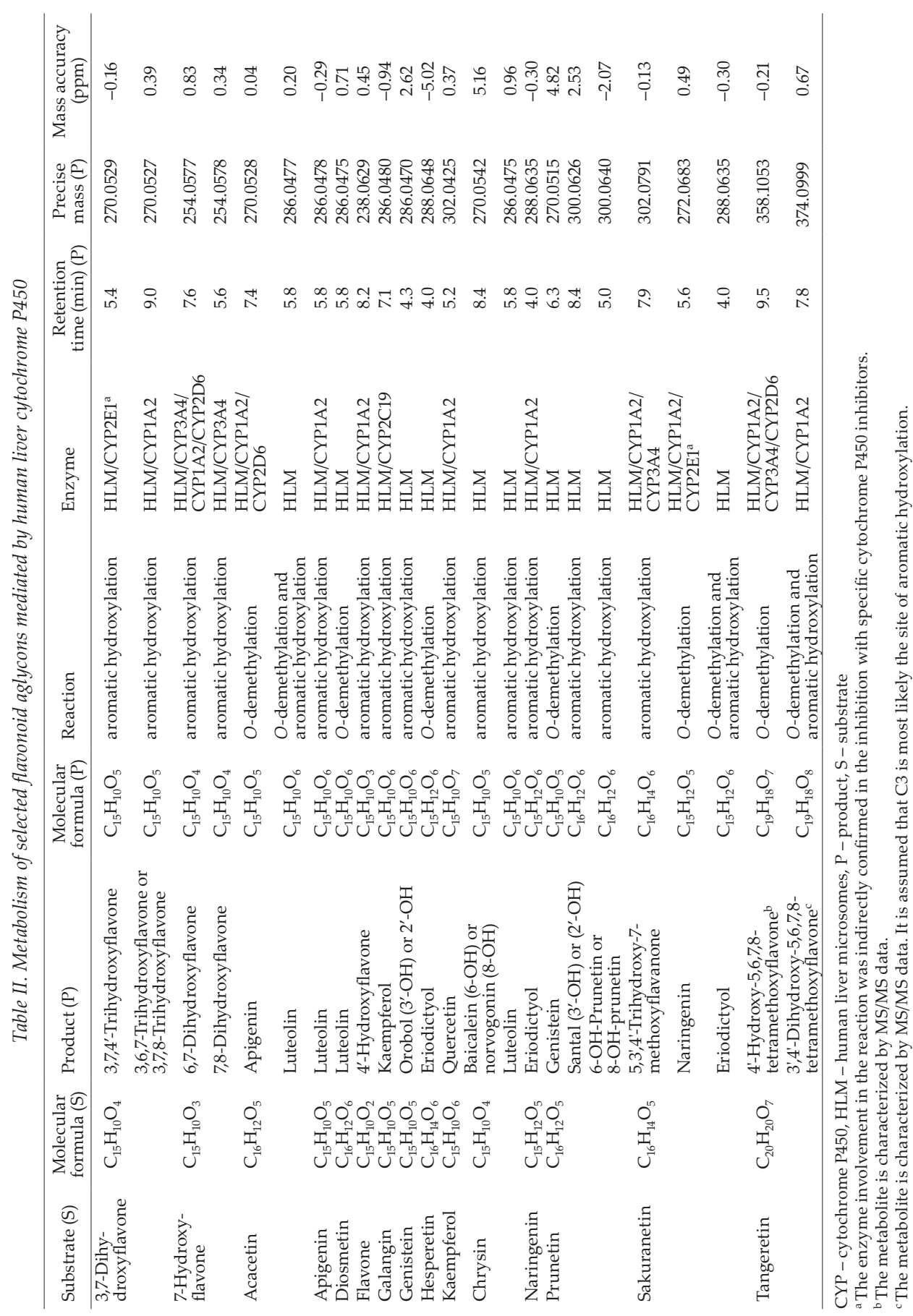


as well as other results presented in this paper, if a flavonoid is already hydroxylated at the position 4', the subsequent aromatic hydroxylation will occur at the position $3^{\prime}$ of the ring B. Therefore, the metabolite at $t_{\mathrm{R}}=7.83 \mathrm{~min}$ was characterized as the most probable $4^{\prime}$-demethylated and 3'-hydroxylated tangeretin derivative, i.e., 3',4'-dihydroxy-5,6,7,8-tetramethoxyflavone. This metabolite is most likely sequentially formed by aromatic hydroxylation at the position $3^{\prime}$ of the ring $\mathrm{B}$ after primary $\mathrm{O}$-demethylation at the position $4^{\prime}$ of the ring $B$.

\section{Determination of cytochromes P450 involved in the metabolism of flavonoid aglycons}

Flavonoids that underwent metabolic changes in human liver microsomes (HLM) incubations were further investigated in incubations with individual recombinant cytochromes P450. The experiments using recombinant cytochromes $\mathrm{P} 450$ were only conducted for those flavonoids that showed metabolite formation in the amount of at least $10 \%(\mathrm{~m} / \mathrm{m})$ compared to the amount of substrate in the HLM experiments. Therefore, the metabolism mediated by individual recombinant cytochrome P450 is reported for the following 10 flavonoid aglycons: 3,7-dihydroxyflavone, 7-hydroxyflavone, acacetin, apigenin, flavone, galangin, kaempferol, naringenin, sakuranetin and tangeretin (Table II).

Incubations with individual recombinant cytochrome P450s indicate that the 3,7-dihydroxyflavone metabolism is mediated by CYP1A2 enzyme. This enzyme was the only one involved in the metabolic conversion of 3,7-dihydroxyflavone but did not catalyze the major metabolic reaction of aromatic hydroxylation at the $4^{\prime}$ position of the B ring observed in the HLM experiments (Fig. 2a), rather the secondary hydroxylation reaction on the ring A at positions 6 or 8, producing the only metabolite 3,6,7-trihydroxyflavone or 3,7,8-trihydroxyflavone $\left(t_{\mathrm{R}}=8.95 \mathrm{~min}\right)$. The major metabolic conversion of 3,7-dihydroxyflavone to 3,7,4'-trihydroxyflavone was not catalyzed by any of the enzymes tested.

7-Hydroxyflavone metabolism (Fig. 2b) is mediated by CYP1A2, CYP2D6 and CYP3A4. Both hydroxylated metabolites (6,7-dihydroxyflavone and 7,8-dihydroxyflavone), observed in the HLM experiment, were generated only by CYP3A4. In the incubations with CYP1A2 and CYP2D6, only 6,7-dihydroxyflavone was detected, the major metabolite which was found in the HLM experiments. Although CYP3A4 catalyzed both metabolic pathways observed in the HLM experiment, the overall contribution is smaller than that of CYP1A2 and CYP2D6. Based on these results, it can be concluded that all three cytochrome P450 enzymes are equally important for the oxidative metabolism of 7-hydroxyflavone.

Acacetin biotransformation (Fig. 2c) is mediated by CYP1A2 and CYP2D6 enzymes. In the experiment with CYP1A2, both metabolic products were observed similarly to the HLM incubations; namely, apigenin and luteolin, while CYP2D6 catalyzed only the demethylation of acacetin producing apigenin. In both experiments, the main product of apigenin was generated in approximately equal amounts.

Apigenin metabolism (Fig. 2c) is mediated exclusively by CYP1A2 enzyme. Just as in the experiments with HLM, incubations with CYP1A2 resulted in the formation of luteolin, suggesting that this enzyme catalyzes the aromatic hydroxylation of apigenin at the position $3^{\prime}$ of the ring B.

Incubations with individual recombinant cytochrome P450s indicate that the flavone (Fig. 2e) metabolism is mediated by the CYP1A2 enzyme. Similarly to the HLM experiment, the dominant metabolite was 4 '-hydroxyflavone $\left(t_{R}=8.59 \mathrm{~min}\right)$. Several other hydroxylated 
products with different elution times were detected using MS detector, but in negligible quantities. This also applies to the peak at $t_{\mathrm{R}}=6.76 \mathrm{~min}$ on the chromatogram which was characterized as a double hydroxylated flavone derivative. The finding of 4'-hydroxyflavone as the main product in CYP1A2 incubation suggests that aromatic hydroxylation of the flavone substrate at the $4^{\prime}$ position of the B ring is catalyzed by this enzyme.

CYP2C19 and CYP2D6 enzymes were found to be responsible for galangin metabolism (Fig. 2f). Just as in the experiments with HLM, incubations of CYP2C19 and CYP2D6 showed the formation of one metabolite - kaempferol, suggesting that this enzyme catalyzes the aromatic hydroxylation of galangin at the position $4^{\prime}$ of the ring B. It should be noted that in both experiments, small amounts of kaempferol were produced in both cases, much less than in HLM experiments. Kaempferol is metabolized by CYP1A2 enzyme (Fig. 2i). In incubations with CYP1A2, similar to the HLM experiment, the only detected metabolite was quercetin $\left(t_{\mathrm{R}}=5.60 \mathrm{~min}\right)$.

Incubations with individual recombinant cytochrome P450s indicate that the metabolism of naringenin is mediated by CYP1A2 enzyme (Fig. 2k). In the incubations with CYP1A2, similar to the HLM experiment, the only detected metabolite was hydroxylated eriodictyol $\left(t_{\mathrm{R}}=3.90 \mathrm{~min}\right)$.

CYP1A2 and CYP3A4 were found to be major enzymes responsible for the metabolism of the sakuranetin (Fig. 2m). Two metabolites were detected in CYP1A2 incubations: $5,3^{\prime} 4^{\prime}$-trihydroxy-7-methoxyflavanone $\left(t_{\mathrm{R}}=7.77 \mathrm{~min}\right)$ and naringenin $\left(t_{\mathrm{R}}=5.53 \mathrm{~min}\right)$. The finding of these two metabolites suggests that CYP1A2 catalyzes the conversion of sakuranetin through $\mathrm{O}$-demethylation and aromatic hydroxylation as determined by the HLM experiments, but their ratios suggest that, unlike the HLM experiment, pure enzyme CYP1A2 favored aromatic hydroxylation of the sakuranetin at the position $3^{\prime}$ of the ring $\mathrm{B}$ when compared to $\mathrm{O}$-demethylation at the position 7 of the ring $\mathrm{A}$. In the incubations with CYP3A4 two metabolites were also detected: 5,3', $4^{\prime}$-trihydroxy-7-methoxyflavanone at $t_{\mathrm{R}}=7.75 \mathrm{~min}$, and an unknown compound with mass difference of $-2,0155 \mathrm{~g} \mathrm{~mol}^{-1}$ compared to the substrate sakuranetin, and molecular formula $\mathrm{C}_{16} \mathrm{H}_{12} \mathrm{O}_{5}\left(t_{\mathrm{R}}=11.74 \mathrm{~min}\right)$. Moreover, this was the predominant metabolic product in CYP3A4 incubations, while the 3'-hydroxylated derivative of sakuranetin was produced in a somewhat smaller amount. Aromatic hydroxylation of sakuranetin at the position $3^{\prime}$ of the ring B is favored compared to the $O$-demethylation at position 7 of the ring A by both CYP3A4 and CYP1A2. The latter metabolic pathway was not catalyzed by CYP3A4 at all. It has to be noted that the sequential aromatic hydroxylation of naringenin, the primary metabolite of sakuranetin in the HLM experiments, and its conversion to the eriodictyol was not catalyzed by any of the enzymes tested.

Tangeretin metabolism was mediated by CYP1A2, CYP2D6, and CYP3A4 enzymes (Fig. 2n). Based on the amount of metabolite produced, it can be concluded that the most important enzymes are CYP1A2 and CYP3A4. However, the role of CYP1A2 is more important as it catalyzes tangeretin's major metabolic reaction observed in the HLM experiment, i.e., 4'-demethylation followed by hydroxylation at the position $3^{\prime}$ of the ring $\mathrm{B}\left(t_{\mathrm{R}}=\right.$ $7.81 \mathrm{~min})$. CYP3A4 catalyzed only the reaction of $4^{\prime}$-demethylation of tangeretin $\left(t_{\mathrm{R}}=9.40\right.$ min), without further conversion to the $3^{\prime}$-hydroxylated product. However, the 4'-demethylated derivative was just the second most commonly found metabolite with CYP3A4, while the major metabolite was a single-demethylated derivative of tangeretin at the ring $\mathrm{A}\left(t_{\mathrm{R}}=10.85 \mathrm{~min}\right)$. CYP2D6 catalyzed only the conversion of tangeretin to the 4 '-demethylated derivative $\left(t_{\mathrm{R}}=9.46 \mathrm{~min}\right)$ in smaller amounts. 

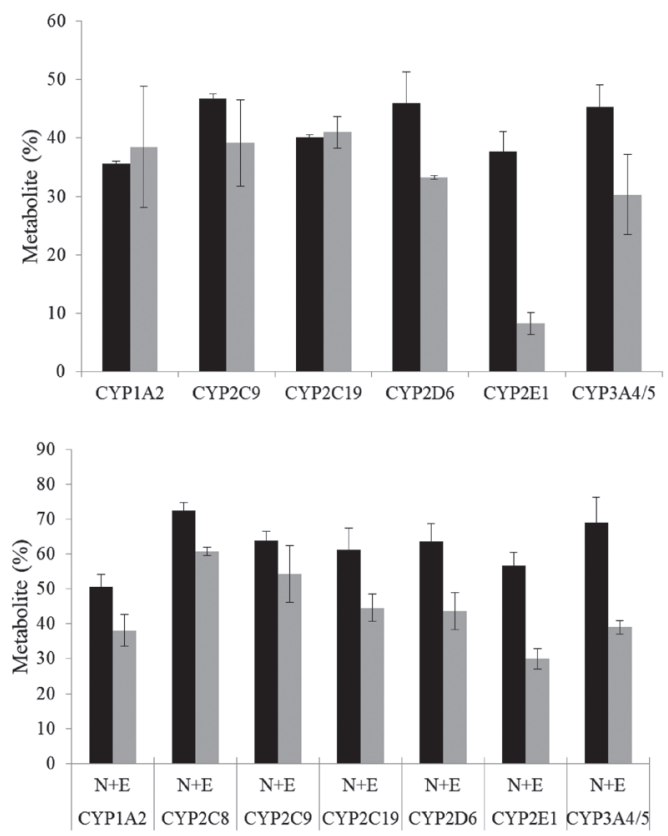

Fig. 4. Inhibition assays for the reactions: a) 3,7-dihydroxyflavone $\rightarrow 3,7,4$-trihydroxyflavone and b) sakuranetin $\rightarrow$ naringenin $(\mathrm{N})+$ eriodictyol (E). Black columns represent the percentage of metabolite/s generated in control without the specific inhibitor of cytochrome P450 and grey columns with the addition of the inhibitor. Results represent the mean \pm standard error $(n=3)$.

None of the recombinant cytochromes $\mathrm{P} 450$ used in the present study catalyzed biotransformation of 3,7-dihydroxyflavone and sakuranetin. As the metabolism of 3,7-dihydroxyflavone and sakuranetin was observed in experiments with the human liver microsomes, further experiments were conducted on the human liver microsomes using specific inhibitors of cytochromes $\mathrm{P} 450$. The reaction of biotransformation of 3,7-dihydroxyflavone to 3,7,4'-trihydroxyflavone was inhibited in the incubations with the specific CYP2E1 inhibitor (4-methylpyrazole, $80 \%$ inhibition, $p<0.05$ ) (Fig. 4).

In the experiment with sakuranetin, the inhibition of the combined reaction of $O$-demethylation of sakuranetin at the 7-position of ring A and of the aforementioned aromatic hydroxylation resulting in naringenin production was observed, when specific inhibitors of cytochromes P450 were used. The observed amounts of metabolites produced were determined by the UV detector. The reaction can be schematically written as: sakuranetin $\rightarrow$ naringenin $\rightarrow$ eriodictyol (Fig. $2 \mathrm{~m}$ ). Obtained results indicate a moderate and statistically significant $(p<0.05)$ inhibition of observed metabolic response in incubations with the specific CYP2E1 inhibitor. Moderate inhibition of $15 \%$ was observed in incubations with the specific CYP2C8 inhibitor (quercetin, $p<0.05$ ) for the total amount of metabolites produced, but not for individual metabolites. Given the very slight inhibition indicated by the results for CYP2C8 and the fact that incubations with the recombinant enzyme did not indicate the metabolism of sakuranetin, this enzyme cannot be regarded as the most important 
one for the metabolism of sakuranetin. However, it can be concluded that among the examined cytochromes P450, CYP2E1 enzyme is only important for catalyzing the observed reaction of sakuranetin $\rightarrow$ naringenin $\rightarrow$ eriodictyol, although the inhibition of $50 \%$ suggests that there could be other cytochromes P450 that may be, to a certain extent, responsible for this metabolic conversion (not analyzed in this study).

\section{Metabolism of flavones}

Acacetin undergoes demethylation and hydroxylation reactions in which CYP1A2 and CYP2D6 enzymes are involved. Androutsopoulos et al. (6) conducted experiments on recombinant CYP1A1 and CYP1B1 enzymes. These enzymes have been investigated primarily because of their potential role in carcinogenesis, but their share in healthy liver metabolism of xenobiotics is virtually negligible (28). Because of all this, it can be said that our study is the first one to describe the metabolism of acacetin mediated by cytochromes P450, which have a relevant meaning for the general population. The results of our study indicate that the oxidative metabolism of acacetin is characterized by two metabolic reactions in which two metabolites are formed: apigenin and luteolin. This is a combined reaction of $O$-demethylation followed by sequential aromatic hydroxylation and can be schematically shown as follows: acacetin $\rightarrow$ apigenin $\rightarrow$ luteolin (Fig. 2c). A complete combined reaction is catalyzed by human liver microsomes and CYP1A2, while CYP2D6 catalyzes only the reaction of demethylation of acacetin to produce apigenin. The results of our study suggest that oxidative metabolism of apigenin is characterized by an aromatic hydroxylation in which luteolin metabolite is formed. This reaction is catalyzed by human liver microsomes and CYP1A2. Androutsopoulos et al. (6) determined the same type of metabolic reaction of apigenin using the CYP1A1 and CYP1B1 enzymes. Although two products were detected in their study, only one was characterized as scutellarein. Scutellarein is the product of the aromatic hydroxylation at position 3 of apigenin, and it was not detected in the herein presented study. Lack of scutellarein as a metabolic product can be attributed to cytochromes P450 used in research due to different objectives of the studies (enzymes involved in carcinogenesis versus enzymes expressed in healthy population; vide supra).

Diosmetin undergoes $\mathrm{O}$-demethylation reaction at position $4^{\prime}$ of the ring B generating luteolin (Fig. 2d). This reaction is catalyzed by human liver microsomes (HLM), and since less than $10 \%(\mathrm{~m} / \mathrm{m})$ of the metabolite is produced in comparison to the substrate concentration, the metabolism of diosmetin was not further characterized. The results obtained in this study agree with those previously published by Androutsopoulos et al. who conducted in vitro experiments with recombinant cytochrome P450 and MCF-7 breast cancer cell lines (10) as well as cell lines of breast adenocarcinoma MDA-MB 468 and normal breast tissue MCF-10A (29) showing that the main route of diosmetin metabolism was the same as suggested in this study. Authors of aforementioned studies $(10,29)$ further determined that CYP2A6, CYP2B6, CYP2C8, CYP2C9, CYP2C19, CYP2D6, CYP2E1, and CYP3A5 are not responsible for this reaction and that the major role in the oxidative metabolism of diosmetin is attributed to CYP1A2 and, to a lesser extent, CYP3A4.

Oxidative metabolism of flavone is characterized by aromatic hydroxylation that generates a metabolite, identified as 4'-hydroxyflavone (Fig. 2e). Minor products of aromatic hydroxylation were detected only by MS detector, but in relatively negligible quantities. The finding of 4'-hydroxyflavone as the main metabolite product suggests that aromatic hydroxylation 
of unsubstituted flavone is mediated by a cytochrome P450 enzyme, namely, CYP1A2. Metabolism of flavone has not been described in the available literature so far, so the results obtained in this paper are the first reported data on its metabolism.

Metabolism of 7-hydroxyflavone is characterized by two metabolic reactions in which two metabolites are formed: 6,7-dihydroxyflavone and 7,8-dihydroxyflavone (Fig. 2a). These are hydroxylated metabolites generated in incubations with human liver microsomes and CYP3A4. In the incubations with CYP1A2 and CYP2D6, only 6,7-dihydroxyflavone, the major metabolite in the HLM experiments, was detected. Although CYP3A4 catalyzed both metabolic pathways observed in the HLM experiment, the overall contribution to the metabolism was somewhat lower than that of CYP1A2 and CYP2D6. The metabolism of 7-hydroxyflavone has not been described in the available literature so far, so the results obtained in this paper are the first report on its metabolism.

Chrysin undergoes two oxidative reactions in which two metabolites are formed: baicalein and luteolin (Fig. 2j). Baicalein is generated by the aromatic hydroxylation at the position 6 of the ring A, and dual aromatic hydroxylation at the positions $3^{\prime}$ and $4^{\prime}$ of the ring B results in the formation of luteolin. This reaction is catalyzed by HLM, and since less than $10 \%$ $(\mathrm{m} / \mathrm{m})$ of metabolites are produced compared to the substrate concentration, the chrysin metabolism was not further characterized.

Most of the detected tangeretin metabolites in human liver microsomes incubations could not be identified due to the lack of appropriate standards. The majority of detected metabolites were single or double demethylated tangeretin derivatives. As tangeretin possesses five methoxy groups, different methoxy groups could undergo demethylation reactions, resulting in various metabolites all of which were detected in smaller amounts. However, the two main metabolites, most commonly found in tangeretin incubations with HLM, were indirectly identified on the basis of data obtained by LC-MS/MS analysis. These were 4'-hydroxy-5,6,7,8-tetramethoxyflavone and 3',4'-dihydroxy-5,6,7,8-tetramethoxyflavone. Major metabolites were formed sequentially by primary $\mathrm{O}$-demethylation at the position $4^{\prime}$ of the ring B followed by aromatic hydroxylation at the position $3^{\prime}$ of the ring B: tangeretin $\rightarrow$ 4'-hydroxy-5,6,7,8-tetramethoxyflavone $\rightarrow$ 3', $4^{\prime}$-dihydroxy-5,6,7,8-tetramethoxyflavone (Fig. 2n). Incubations with individual recombinant cytochrome P450s indicate that tangeretin metabolism is mediated by CYP1A2, CYP2D6, and CYP3A4 enzymes. Based on the amount of metabolites produced, it can be concluded that the most important role is attributed to CYP1A2 and CYP3A4. CYP1A2 is the most important enzyme for the oxidative metabolism of tangeretin since it catalyzed its major metabolic reaction observed in the HLM experiments. The results obtained are well related to the already published tangeretin metabolism data (Table I). The main metabolite with the highest content, in this case, was $4^{\prime}$-hydroxy-5,6,7,8-tetramethoxyflavone. Experiments with recombinant cytochrome P450 showed that enzymes CYP1A2, CYP3A4, and CYP2D6 were involved in the oxidative metabolism of tangeretin, but it was not accurately determined which enzyme was responsible for each individual metabolic reaction. As in this study, it has been shown that the major enzyme CYP1A2 is responsible for demethylation at the position $4^{\prime}$ of the ring B, as well as post hydroxylation at position $3^{\prime}$ of the ring B of the demethylated product. The same biotransformation was observed with CYP3A4, but to a lesser extent. The results for CYP3A4 are partially in discrepancy with the fact that no further oxidation of the 4 '-demethylated product in CYP3A4 incubations has been observed in this study. However, Breinholt et al. (12) observed oxidation of the 4'-demethylated product in low quantities. This minor difference 
between the herein presented and literature data could be attributed to differences in experimental conditions. It is particularly important to note that the quantity of cytochrome P450 used in their study is not disclosed. Finally, we can conclude that the results presented herein confirmed the main route of tangeretin metabolism mediated by human liver cytochrome P450 and the major role of CYP1A2 in this biotransformation.

\section{Metabolism of flavonols}

In this paper 10 flavonoids belonging to the flavonol group were tested. For seven of them, information about metabolism can be found in the literature (galangin, isorhamnetin, kaempferol, quercetin, myricetin, morin, and tamarixetin) (Table I). For the remaining three tested flavonoids from this group (3,6-dihydroxyflavone, 3,7-dihydroxyflavone, and rhamnetin), this is the first report on cytochrome P450 mediated metabolism. Metabolism mediated by human liver cytochrome P450 under experimental conditions described in this paper was not recorded for the following flavonols: 3,6-dihydroxyflavone, isorhamnetin, quercetin, myricetin, morin, rhamnetin, and tamarixetin. This result confirmed data published by Nielsen et al. (7) who also determined the absence of metabolism of isorhamnetin, quercetin, myricetin, and morin in incubation with rat cytochromes $\mathrm{P} 450$.

While in our study tamarixetin metabolism was not detected, its metabolism was noted in the previous studies by Nielsen et al. (7) and Breinholt et al. (8) who determined the metabolic conversion of tamarixetin into quercetin by the reaction of demethylation at the position $4^{\prime}$ of the ring B mediated by the enzyme CYP1A2. When comparing the enzyme systems, HLM and recombinant cytochromes P450, two evident differences in the studies have been noticed. Breinholt et al. (8) used markedly greater enzyme concentration (up to 30-fold) relative to incubation in our study. At the same time concentrations of the enzyme in human liver microsomes incubations were not reported.

Incubations with individual recombinant cytochrome P450s indicate that the 3,7-dihydroxyflavone metabolism is mediated by CYP1A2 enzyme. This enzyme was the only tested enzyme involved in the metabolic conversion of 3,7-dihydroxyflavone but it did not catalyze the major metabolic reaction - aromatic hydroxylation at the $4^{\prime}$ position of the B ring observed in the HLM experiments. However, CYP1A2 did catalyze the secondary hydroxylation reaction on the ring A at the positions 6 or 8, forming only one metabolite, 3,6,7-trihydroxyflavone or 3,7,8-trihydroxyflavone. The major metabolic conversion of 3,7-dihydroxyflavone to 3,7,4'-trihydroxyflavone was not catalyzed by any of the enzymes tested. Based on the results of the inhibition assays with specific cytochrome P450 inhibitors, the major reaction was attributed to the CYP2E1 enzymes. The results of the 3,7-dihydroxyflavone metabolism are particularly interesting because it is the only example in which purified enzyme (CYP1A2) catalyzed the secondary reaction to a greater extent when compared to the major reaction catalyzed by HLM.

Oxidative metabolism of galangin is characterized by a metabolic reaction in which a kaempferol metabolite is formed. This reaction is catalyzed by human liver microsomes, CYP2C19 and CYP2D6. Under the experimental conditions, small amounts of kaempferol were produced in pure enzyme incubations, much less when compared to the HLM experiment. Otake and Walle (13) found that CYP1A2 had a dominant role in this metabolic conversion and CYP2C9 to some extent. Based on the small differences in the experiment conditions, it is not possible to find a clear explanation for the observed difference when comparing the results to our study. Otake and Walle (13) investigated galangin metabolism 
with three relevant recombinant pure enzymes CYP1A2, CYP2C9, and CYP3A4. Although the main role of CYP1A2 has been found, based on inhibition assays using specific inhibitors where the metabolism mediated by HLM was inhibited by $40 \%$ with a specific CYP1A2 inhibitor, the authors determined that other specific cytochrome P450 enzymes may be involved in this metabolism as well.

Kaempferol metabolism is characterized by aromatic hydroxylation at the position $3^{\prime}$ of the ring B in which quercetin is formed. This metabolic conversion was catalyzed by human liver microsomes and CYP1A2. Kaempferol metabolism has been described in the literature in the work of Breinholt et al. (8) and the results of our study are fully consistent with these results.

\section{Metabolism of isoflavones}

In this study, two representatives of isoflavones were tested: genistein and prunetin. For both flavonoids, oxidative metabolism mediated by human cytochrome P450 is well described in the literature and particularly well documented for genistein (Table I).

The results of this study indicate that the oxidative metabolism of genistein is characterized by a single metabolic reaction in which an orobol metabolite is formed by aromatic hydroxylation at the position $3^{\prime}$ of the ring B. This reaction is catalyzed by human liver microsomes (HLM), and since less than $10 \%(\mathrm{~m} / \mathrm{m})$ of metabolites is produced in comparison to the substrate concentration, the metabolism of genistein was not further characterized. The results obtained in this study agree with the previously published literature; the aforementioned reaction is the dominant reaction reported in the literature (14-19).

Oxidative metabolism of the prunetin is characterized by three metabolic reactions in which the following metabolites are formed: genistein, 6-hydroxyprunetin and 3'-hydroxyprunetin. $\mathrm{O}$-demethylation is a somewhat preferred metabolic pathway of prunetin biotransformation by cytochromes P450. However, it should be noted that the remaining two hydroxylated products were found in similar amounts, which were lower when compared to the amount of genistein. All three reactions are catalyzed by human liver microsomes, to the extent less than $10 \%(\mathrm{~m} / \mathrm{m})$; consequently, the metabolism of the prunetin was not further characterized. The results obtained in this study agree with the previously published research of Hua et al. (17), who described the observed metabolic reaction as a very rapid conversion of prunetin to genistein, but at the same time did not report any other metabolic pathway of prunetin. The largest difference between the two studies was again in the incubation enzyme concentration; Hua et al. used markedly higher concentrations, about 3 times greater than in the HLM experiments in our study.

\section{Metabolism of flavanones}

In this study, six flavonoids belonging to the flavanone group were subjected to cytochrome P450 catalyzed reactions. In the primary scientific literature, only hesperetin and naringenin metabolism is described to some extent $(7,8,20)$. For the remaining four examined flavonoids from this group (flavanon, pinocembrin, pinocembrin-7-methylether and sakuranetin), this is the first report on cytochrome P450 mediated metabolism.

Hesperetin undergoes $O$-demethylation reaction at the position $4^{\prime}$ of the ring $\mathrm{B}$ generating eriodictyol. This reaction is catalyzed by human liver microsomes to a lesser extent 
(less than $10 \%, \mathrm{~m} / \mathrm{m}$ ) and was not further characterized. The results obtained in this study agree with those previously published by Nielsen $e t$ al. (7) and Breinholt et al. (8) who conducted incubations with rat liver microsomes, HLM and recombinant CYP1A2 showing that the major route of hesperetin metabolism was identical to the one suggested in our research. Authors of the aforementioned studies $(7,8)$ also determined that CYP2C9, CYP2D6 and CYP3A4 are not responsible for this reaction, and that the major role in hesperetin oxidative metabolism is attributed to CYP1A2. These two studies as well as ours are contrary to the results of Doostdar et al. (20) who did not detect metabolism of hesperetin mediated by cytochromes P450, which is most likely due to too low concentrations of flavonoids used

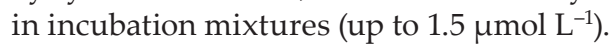

Naringenin is biotransformed to eriodictyol in the reaction of aromatic hydroxylation at the position $3^{\prime}$ of the ring B. This reaction was observed in experiments with human liver microsomes, and attributed to CYP1A2. The result of the metabolism of naringenin obtained in this study agrees with the literature data with regard to the reaction catalyzed by human liver cytochrome P450, in which the main metabolite produced is eriodictyol. Conclusions regarding the specific cytochrome P450 enzymes dominantly responsible for the catalysis of this reaction are, however, to some extent different from those published. While Breinholt et al. (8) found that CYP1A2 had a dominant role in this metabolic conversion, our study showed that CYP3A4 did not metabolize naringenin under experimental conditions and that CYP1A2 was only a secondary enzyme responsible for the observed naringenin metabolism. The enzyme dominantly responsible for this metabolic reaction was not found and was not among the tested group of enzymes (CYP1A2, CYP2A6, CYP2C8, CYP2C9, CYP2C19, CYP2D6 and CYP3A4).

The results of the herein presented study suggest that there are two metabolic pathways of sakuranetin biotransformation mediated by the human liver cytochromes P450. Sakuranetin is metabolized to naringenin, eriodictyol, and 5,3', '4'-trihydroxy-7-methoxyflavanone; enzymes responsible for the metabolism are CYP1A2 and CYP3A4. In the incuba-

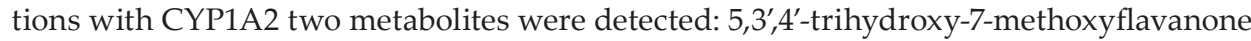
and naringenin. The finding of these two metabolites suggests that CYP1A2 catalyzes the conversion of sakuranetin through $\mathrm{O}$-demethylation and aromatic hydroxylation as determined by the HLM experiments but their ratios suggest that, unlike the HLM experiment, CYP1A2 enzyme favored aromatic hydroxylation of the sakuranetin at the position $3^{\prime}$ of the ring $\mathrm{B} v s$. $\mathrm{O}$-demethylation at the position 7 of the ring $\mathrm{A}$. In the incubations with CYP3A4, two metabolites were detected: again 5,3', $4^{\prime}$-trihydroxy-7-methoxyflavanone and an unknown compound with molecular formula $\mathrm{C}_{16} \mathrm{H}_{12} \mathrm{O}_{5}$. Moreover, 5,3', '-trihydroxy-7-methoxyflavanone was the predominant metabolic product in CYP3A4 incubations, while the $3^{\prime}$-hydroxylated derivative of sakuranetin was produced in a somewhat smaller amount. This metabolite was also found in HLM incubations, but in much smaller amounts compared to other metabolites, suggesting that this third metabolic pathway of sakuranetin metabolism is mediated by cytochromes P450. Interestingly, the sequential aromatic hydroxylation of naringenin, the primary metabolite from the HLM experiment, and its conversion to the eriodictyol was not observed. Inhibition assays with specific cytochrome P450 inhibitors showed that CYP2E1 was the only significant enzyme involved in catalysis of the reaction sakuranetin $\rightarrow$ naringenin $\rightarrow$ eriodictyol. The sakuranetin metabolism has not been described in the available literature so far, so the results obtained in this paper are the first known data on its metabolism. 


\section{CONCLUSIONS}

Hydroxylation of flavones is catalyzed by human cytochrome P450 at position 3' and $4^{\prime}$ of ring B, with position $4^{\prime}$ being favored on unsubstituted ring B. Hydroxylation is also possible at positions 6 and 8 of the ring A. Aromatic hydroxylation of an unsubstituted flavone is preferred at the position $4^{\prime}$ of the ring B, but that hydroxylation is also possible at other positions in the flavone structure. $O$-demethylation of the methoxy group in flavone is favored at position $4^{\prime}$ of ring $\mathrm{B}$, although tangeretin is an example for which demethylation is also possible on ring A. The enzyme dominantly responsible for the observed metabolic reactions of the flavone class is CYP1A2. Although the majority of the flavonoids from flavones class were examined (eleven), half of them did not show oxidative metabolism mediated by cytochromes $\mathrm{P} 450$.

Similarly to flavones, flavonols undergo aromatic hydroxylation catalyzed by human liver cytochrome P450 at the ring B, positions 3' and 4', whereas position 4' is favored on unsubstituted ring B. However, in the case of 3,7-dihydroxyflavone metabolism, it can be seen that hydroxylation is also possible on ring A at positions 6 and 8, position 6 being favored in relation to position 8 . The presence of two or more hydroxyl groups on the B ring of the flavonols appears to prevent further metabolism mediated by human liver cytochromes P450. Although several mono-methylated flavonols representatives were tested, $\mathrm{O}$-demethylation was not observed in this flavonoid class of compounds. The enzyme that is dominantly responsible for the observed flavonols metabolism is CYP1A2, but its role is not expressed to such a degree as in the case of flavones, whereas in the case of 3,7-dihydroxyflavone it is shown that some other specific cytochrome P450 (i.e., CYP2E1) has a more important role in its metabolism.

A sample of the examined isoflavones in this study is too small to extract general conclusions about their oxidative metabolism mediated by human liver cytochrome P450, but it can be noted that results are consistent with the genistein and prunetin data already reported in the literature. Likewise, the observed aromatic hydroxylation at the position 3' of the ring $\mathrm{B}$ of genistein and prunetin fits well into overall results for tested flavonoids, especially for the flavone group structurally closest to isoflavones. Thus, isoflavone ring B hydroxylation is favored at position 3', if a hydroxyl group at position 4 ' is already present in the structure. In the example of prunetin it is shown that $O$-demethylation is possible at position 7 of the ring $\mathrm{A}$ and that aromatic hydroxylation is possible at position 6 of the ring A in isoflavone skeleton.

Flavanone results were in good agreement with those reported for hesperetin and naringenin. The observed aromatic hydroxylation at the position 3' of the ring B of naringenin and sakuranetin fits well with the overall results for tested flavonoids. Flavanone undergoes hydroxylation of the ring B that is favored at the position 3', if a hydroxyl group at the position $4^{\prime}$ is already present in the structure. The importance of this hydroxyl group for the oxidative metabolism of flavanone is further underlined by the absence of metabolism of flavanone pinocembrin and pinocembrin-7-methylate having an unsubstituted ring $\mathrm{B}$. In the example of hesperetin and sakuranetin it is shown that $O$-demethylation is possible at the position $4^{\prime}$ of the ring $\mathrm{B}$ and at the position 7 of the ring A. CYP1A2 is dominantly responsible for observed flavanone metabolism, whereas other specific cytochrome P450 (i.e., CYP2E1 or CYP3A4) plays an important role in the metabolism of sakuranetin and naringenin. 
The overall conclusion of this study is that flavonoid aglycons undergo two types of oxidative reactions: aromatic hydroxylations and $O$-demethylations catalyzed mainly by cytochrome P450 1A2. Aromatic hydroxylation is favored at the position 4' of B ring, and in $3^{\prime}$ if a hydroxyl group at the position $4^{\prime}$ is already present in the structure. Moreover, the presence of two or more hydroxyl groups on the B ring of the flavanols appears to prevent further metabolism mediated by human liver cytochromes P450. O-demethylation of the methoxy group is favored at position 4' of ring B. These results, to some extent, contribute to the understanding of the metabolism of constituents found in antioxidant dietary supplements and their possible interactions with other xenobiotics, i.e., interactions with drugs metabolized by the same cytochromes $\mathrm{P} 450$ as flavonoids.

Acknowledgements. - This research was supported by the Croatian Science Foundation under the project UIP-2014-09-5704 (M. B.). The authors thank anonymous reviewers, whose suggestions have improved the manuscript.

\section{REFERENCES}

1. J. A. Yáñez, P. K. Andrews and N. M. Davies, Methods of analysis and separation of chiral flavonoids, J. Chromatogr. B 848 (2007) 159-181; https://doi.org/10.1016/j.jchromb.2006.10.052

2. J. Xiao and P. Högger, Metabolism of dietary flavonoids in liver microsomes, Curr. Drug Metab. 14 (2013) 381-391; https://doi.org/10.2174/1389200211314040003

3. B. H. Havsteen, The biochemistry and medical significance of the flavonoids, Pharmacol. Therapeut. 96 (2002) 67-202; https://doi.org/10.1016/S0163-7258(02)00298-X

4. J. Viskupičová, M. Ondrejovič and E. Šturdík, Bioavailability and metabolism of flavonoids, J. Food Nutr. Res. 47 (2008) 151-162.

5. F. P. Guengerich, Cytochrome P450 and chemical toxicology, Chem. Res. Toxicol. 21 (2008) 70-83; https:// doi.org/10.1021/tx700079z

6. V. P. Androutsopoulos, A. Papakyriakou, D. Vourloumis and D. A. Spandidos, Comparative CYP1A1 and CYP1B1 substrate and inhibitor profile of dietary flavonoids, Bioorg. Med. Chem. 19 (2011) 28422849; https://doi.org/10.1016/j.bmc.2011.03.042

7. S. E. Nielsen, V. Breinholt, U. Justesen, C. Cornett and L. O. Dragsted, In vitro biotransformation of flavonoids by rat liver microsomes, Xenobiotica 28 (1998)389-401; https://doi.org/10.1080/004982598239498

8. V. M. Breinholt, E. A. Offord, C. Brouwer, S. E. Nielsen, K. Brøsen and T. Friedberg, In vitro investigation of cytochrome P450-mediated metabolism of dietary flavonoids, Food Chem. Toxicol. 40 (2002) 609-616; https://doi.org/10.1016/S0278-6915(01)00125-9

9. A. Gradolatto, M. C. Canivenc-Lavier, J. P. Basly, M. H. Siess and C. Teyssier, Metabolism of apigenin by rat liver phase I and phase II enzymes and by isolated perfused rat liver, Drug Metab. Dispos. 32 (2004) 58-65; https://doi.org/10.1124/dmd.32.1.58

10. V. Androutsopoulos, N. Wilsher, R. R. J. Arroo and G. A. Potter, Bioactivation of the phytoestrogen diosmetin by CYP1 cytochromes P450, Cancer Lett. 274 (2009) 54-60; https://doi.org/10.1016/j.canlet.2008.08.032

11. S. E. Nielsen, V. Breinholt, C. Cornett and L. O. Dragsted, Biotransformation of the citrus flavone tangeretin in rats. Identification of metabolites with intact flavane nucleus, Food Chem. Toxicol. 38 (2000) 739-746; https://doi.org/10.1016/S0278-6915(00)00072-7

12. V. M. Breinholt, S. E. Rasmussen, K. Brøsen and T. H. Friedberg, In vitro metabolism of genistein and tangeretin by human and murine cytochrome P450s, Pharmacol. Toxicol. 93 (2003) 14-22; https://doi. org/10.1034/j.1600-0773.2003.930102.x 
13. Y. Otake and T. Walle, Oxidation of the flavonoids galangin and kaempferide by human liver microsomes and CYP1A1, CYP1A2 and CYP2C9, Drug Metab. Dispos. 30 (2002) 103-105; https://doi.org/10.1124/ dmd.30.2.103

14. S. E. Kulling, D. M. Honig and M. Metzler, Oxidative metabolism of the soy isoflavones daidzein and genistein in humans in vitro and in vivo, J. Agric. Food Chem. 49 (2001) 3024-3033; https://doi.org/10.1021/ jf0012695

15. S. E. Kulling, D. M. Honig, T. J. Simat and M. Metzler, Oxidative in vitro metabolism of the soy phytoestrogens daidzein and genistein, J. Agric. Food Chem. 48 (2000) 4963-4972; https://doi.org/10.1021/ jf000524i

16. S. E. Kulling, L. Lehmann and M. Metzler, Oxidative metabolism and genotoxic potential of major isoflavone phytoestrogens, J. Chromatogr. B 777 (2002) 211-218; https://doi.org/10.1016/S1570-0232(02)00215-5

17. M. Hu, K. Krausz, J. Chen, X. Ge, J. Li, H. L. Gelboin and F. J. Gonzalez, Identification of CYP1A2 as the main isoform for the phase I hydroxylated metabolism of genistein and a prodrug converting enzyme of methylated isoflavones, Drug Metab. Dispos. 31 (2003) 924-931; https://doi.org/10.1124/dmd.31.7.924

18. J. Bursztyka, E. Perdu, J. Tulliez, L. Debrauwer, G. Delous, C. Canlet, G. De Sousa, R. Rahmani, E. Benfenati and J. P. Cravedi, Comparison of genistein metabolism in rats and humans using liver microsomes and hepatocytes, Food Chem. Toxicol. 46 (2008) 939-948; https://doi.org/10.1016/j.fct.2007.10.023

19. K. M. Atherton, E. Mutch and D. Ford, Metabolism of the soyabean isoflavone daidzein by CYP1A2 and the extra-hepatic CYPs 1A1 and 1B1 affects biological activity, Biochem. Pharmacol. 72 (2006) 624-631; https://doi.org/10.1016/j.bcp.2006.05.015

20. H. Doostdar, M. D. Burke and R. T. Mayer, Bioflavonoids: selective substrates and inhibitors for cytochrome P450 CYP1A and CYP1B1, Toxicology 144 (2000) 31-38; https://doi.org/10.1016/S0300483X(99)00215-2

21. J. M. Young, W. Xiaodong and E. M. Marilyn, Dietary flavonoids: Effects on xenobiotic and carcinogen metabolism, Toxicol. In Vitro 20 (2006) 187-210; https://doi.org/10.1016/j.tiv.2005.06.048

22. Y. Kimura, H. Ito, R. Ohnishi and T. Hatano, Inhibitory effects of polyphenols on human cytochrome P450 3A4 and 2C9 activity, Food Chem. Toxicol. 48 (2010) 429-435; https://doi.org/10.1016/j.fct.2009.10.041

23. M. Bojić, C. A. Sedgeman, L. D. Nagy and F. P. Guengerich, Aromatic hydroxylation of salicylic acid and aspirin by human cytochromes P450, Eur. J. Pharm. Sci. 73 (2015) 49-56; https://doi.org/10.1016/j. ejps.2015.03.015

24. M. Medić-Šarić, V. Rastija and M. Bojić, Recent advances in the application of high performance liquid chromatography in the analysis of polyphenols in wine and propolis, J. AOAC Int. 94 (2011) 32-42.

25. M. Barbarić, K. Mišković, M. Bojić, M. Baus Lončar, A. Smolčić-Bubalo, Ž. Debeljak and M. Medić-Šarić, Chemical composition of the ethanolic propolis extracts and its effect on HeLa cells, J. Ethnopharmacol. 135 (2011) 772-778; https://doi.org/10.1016/j.jep.2011.04.015

26. D. Tsimogiannis, M. Samiotaki, G. Panayotou and V. Oreopoulou, Characterization of flavonoid subgroups and hydroxy substitution by HPLC-MS/MS, Molecules 12 (2007) 593-606; https://doi. org/10.3390/12030593

27. F. Cuyckens and M. Claeys, Mass spectrometry in the structural analysis of flavonoids, J. Mass Spectrom. 39 (2004) 1-15; https://doi.org/10.1002/jms.585

28. F. P. Guengerich, Human Cytochrome P450 Enzymes, in Cytochrome P450: Structure, Mechanism, and Biochemistry (Ed. P. R. Ortiz de Montellano), 4th ed., Springer Int. Publ. Switzerland, Cambridge 2015; https://doi.org/10.1007/978-3-319-12108-6_9

29. V. P. Androutsopoulos, S. Mahale, R. R. J. Arroo and G. Potter, Anticancer effects of the flavonoid diosmetin on cell cycle progression and proliferation of MDA-MB 468 breast cancer cells due to CYP1 activation, Oncol. Rep. 21 (2009) 1525-1528; https://doi.org/10.3892/or_00000384 